\title{
Design of Compact Low-Pass Elliptic Filters Using Double-Sided MIC Technology
}

\author{
Mariá del Castillo Velázquez-Ahumada, Jesús Martel, and Francisco Medina, Senior Member, IEEE
}

\begin{abstract}
A novel implementation of stepped-impedance low-pass elliptic filters is presented in this paper. The filters are based on the well-known technique of cascading high- and low-impedance sections to simulate the ladder $L C$ lumped-circuit prototype. We propose in this study a new approach to build up the constitutive circuit elements by taking advantage of the use of both sides of the substrate. The use of double-sided technology yields both design flexibility and good circuit performance. High-impedance sections are achieved by using slots in the backside of the substrate, whereas low-impedance sections are obtained with parallel-plate capacitors. In order to achieve the transmission poles corresponding to the elliptic design, these capacitors are series connected to the ground plane by means of high-impedance coplanar-waveguide lines, which mainly act as inductors. As a final step, meandering techniques have been applied to the high-impedance sections of the filter to reduce the overall circuit size. The measurement of several fabricated filters shows fairly good agreement between theory and experiment.
\end{abstract}

Index Terms-Double-sided microwave integrated circuit (MIC), elliptic response, low-pass filters (LPFs), steppedimpedance (SI) filters.

\section{INTRODUCTION}

$\mathbf{L}$ OW-PASS filters (LPFs) are very important components used to eliminate unwanted harmonics, as well as spurious bands in microwave and millimeter-wave systems. The conventional stepped-impedance low-pass filter (SI-LPF) consists of a cascading of electrically short high- and low-impedance sections to approximate the corresponding ladder $L C$ lumpedcircuit prototype. SI-LPFs fabricated using conventional microstrip lines are among the most commonly used LPFs owing to simple design and low fabrication cost [1]. However, this type of filter inherently presents two important practical problems. The first one is the degradation of the out-of-band rejection level because of the frequency-distributed behavior of the finite section lines beyond the cutoff frequency. The second problem comes from the limits imposed by the microstrip technology to achieve very narrow strip widths and, consequently, high-impedance section lines.

Manuscript received February, 15 2006; revised September 26, 2006. This work was supported by the Spanish Ministry of Education and Science/European Union under FEDER Funds Project TEC2004-03214 and by the Spanish Junta de Andalucía under Project TIC-253.

M. del Castillo Velázquez-Ahumada and F. Medina are with the Facultad de Física, Grupo de Microondas, Departamento de Electrónica y Electromagnetismo, Universidad de Sevilla, 41012 Seville, Spain (e-mail: velazquez@us.es; medina@us.es).

J. Martel is with the Departamento de Física Aplicada II, Grupo de Microondas, Escuela Técnica Superior de Arquitectura, Universidad de Sevilla, 41012 Seville, Spain (e-mail: martel@us.es).

Digital Object Identifier 10.1109/TMTT.2006.888578
The rejection level in the out-of-band region of the SI-LPF, when conventional Chebyshev or Butterworth implementations are used, can be obviously improved by increasing the number of filter sections. However, it is also evident that this solution implies increasing the circuit size, as well as the bandpass losses. Recently, the use of defected ground structures (DGSs) has been proposed [2], [3] as an efficient method to attain a good out-ofband response of SI-LPFs. Thus, the influence of using periodic DGSs of various shapes on the response of conventional SI-LPF designs is analyzed in [2]. An equivalent circuit for the combination of the line section and DGS is introduced in [3] to design LPFs with different DGSs in each filter section. Alternatively, complementary split-ring resonators (CSRRs) can be etched on the conductor strips of the low-impedance sections to suppress spurious bands in conventional SI-LPF [4]. However, if a sharper cutoff is required for the LPF, generalized Chebyshev [5] or elliptic [6]-[8] designs have to be chosen in order to introduce transmission zeros in the vicinity of the bandpass. A broadband LPF based on a generalized Chebyshev lumped-circuit prototype is designed in [5] making use of open-circuit stubs in suspended substrate stripline technology. Microstrip rectangular elements are cascaded in [6] to obtain a broadband elliptic filter response. Multiple cascaded stepped-impedance hairpin resonators have also been proposed in [7] and [8] in order to obtain a compact elliptic LPF with sharp cutoff frequency response.

In this paper, the design of an elliptic response LPF that retains the simplicity of the stepped-impedance structure is described. The method is based on the implementation of the constituent filter components by properly patterning the metallization at both sides of the substrate. Circuits fabricated using this technology are usually called a double-sided microwave integrated circuit (MIC) [9]. They take advantage of using different transmission media (microstrip, slot line, coplanar waveguide (CPW), microstrip-slot lines) on both sides of the substrate, therefore adding more design flexibility. In Fig. 1, we show an elementary layout of the proposed SI-LPF concept (in this case, a three-pole filter with a single transmission zero) together with its corresponding lumped equivalent circuit. Note that the $L C$ shunt section is implemented in a single stage involving both sides of the substrate. The series inductors $L_{s i}$ have been realized, as usual, by means of electrically short high-impedance transmission-line sections. The slot in the ground plane under the strip line contributes to achieve values of the characteristic impedance higher than those obtained with conventional microstrip lines [10], [11]. As a first-order approximation, lowimpedance sections are assimilated to parallel-plate capacitors to approximate the parallel connected capacitances $C_{p i}$. In order 


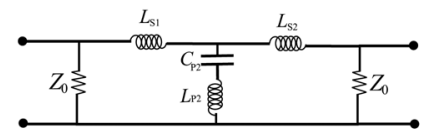

(a)

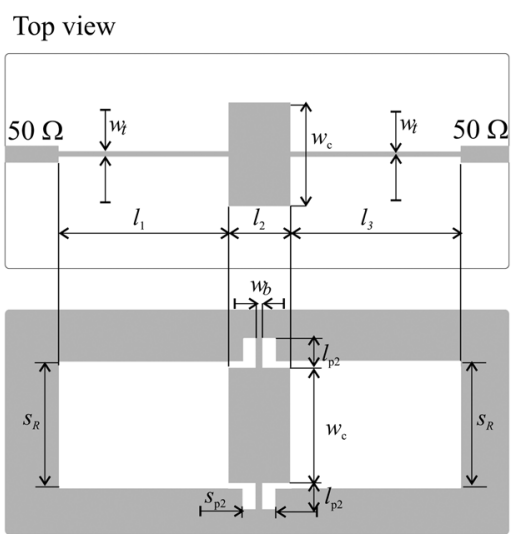

Bottom view

(b)

Fig. 1. (a) Lumped equivalent circuit of a three-pole LPF with a single transmission zero. (b) Layout of the planar circuit implementation of the corresponding SI-LPF proposed in this paper.

to synthesize the transmission poles corresponding to the elliptic design, those capacitors are then series connected to the ground plane through high-impedance CPW line sections, which act as the inductors present in the parallel branches $L_{p i}$. Please note that high-impedance CPW lines are located at opposite borders of the backside rectangular patch of the capacitors; therefore, they play the additional role of bridges joining the two sides of the ground plane at the bottom side of the filter layout. These bridges are essential for a good filter performance because they cancel out undesired ground-plane slot modes [12], [13]. It is worth mentioning here that, owing to their small electrical size, slots in the ground plane do not meaningfully behave as radiant elements in the frequency range of interest, although radiation might be important at higher frequencies.

The geometrical parameters of the layout have been obtained starting from the standard tabulated lumped equivalent-circuit components. We have made use of the formula for the equivalent circuit of electrically short high characteristic impedance transmission-line sections [1] to calculate the length of those sections. The characteristic impedance values have been obtained from the fast quasi-TEM algorithm developed in [14] for the analysis of hybrid microstrip-CPW structures. The lengths of the capacitors have been roughly calculated from the trivial expression for the capacitance of an ideal parallel-plate capacitor without edge effects. Finally, we have made use of a commercial electromagnetic (EM) simulator to take into account these edge effects and to correct the capacitor lengths obtained from the crude parallel-plate capacitance expression. Although the use of slots in the backside of the high-impedance sections leads itself to a size reduction with respect to conventional microstrip implementation [11], we have also used a meander line to achieve more compact filters [18]. In this way, the high-impedance section lines have been substituted by series-connected open loops. The geometry of these open loops has been extracted from EM
TABLE I

SPECIFICATIONS AND VALUES OF THE COMPONENTS OF THE LUMPED-CIRCUIT PROTOTYPE OF FILTER A

Order: $N=3$

Cutoff frequency: $f_{c}=2.3 \mathrm{GHz}$

Passband ripple $L_{A r}=0.1 \mathrm{~dB}$

Stopband starting frequency $f_{s}=5.75 \mathrm{GHz}$

Minimum insertion loss in stopband $L_{A s}=30.5 \mathrm{~dB}$

\begin{tabular}{|c|c|}
\hline Sections 1 and 3 & $L_{s 1}=L_{s 3}=3.28 \mathrm{nH}$ \\
\hline Section2 & $L_{p 2}=0.417 \mathrm{nH}, C_{p 2}=1.41 \mathrm{pF}$ \\
\hline
\end{tabular}

TABLE II

SPECIFICATIONS AND VALUES OF THE COMPONENTS OF THE LUMPED-CIRCUIT PROTOTYPE OF FILTER $B$

Order: $N=5$

Cutoff frequency: $f_{c}=1.8 \mathrm{GHz}$

Passband ripple $L_{A r}=0.1 \mathrm{~dB}$

Stopband starting frequency $f_{s}=3.27 \mathrm{GHz}$

Minimum insertion loss in stopband $L_{A s}=54.0 \mathrm{~dB}$

\begin{tabular}{|c|c|}
\hline Section 1 & $L_{s 1}=4.74 \mathrm{nH}$ \\
\hline Section 2 & $L_{p 2}=0.406 \mathrm{nH}, C_{p 2}=2.25 \mathrm{pF}$ \\
\hline Section 3 & $L_{s 3}=7.75 \mathrm{nH}$ \\
\hline Section 4 & $L_{p 4}=1.13 \mathrm{nH}, C_{p 4}=1.93 \mathrm{pF}$ \\
\hline Section 5 & $L_{s 5}=4.14 \mathrm{nH}$ \\
\hline
\end{tabular}

simulation in such a way that they reproduce the frequency response of the original straight line sections fed by two 50- $\Omega$ transmission lines. Two selected examples are presented to illustrate the design procedure. Experimental confirmation of the theory is also provided.

\section{FILTER DESIGN}

In order to illustrate the use of the proposed structure and the design procedure, we have designed a couple of elliptic LPFs of orders $N=3$ and $N=5$. The filter specifications and the corresponding lumped parameter values are shown in Tables I and II. These values can be easily computed from the tabulated element values for elliptic function low-pass prototype filters [1] after applying impedance $(50 \Omega)$ and frequency scaling. Our purpose is to design and fabricate the filters $A$ and $B$ using a commercial substrate of relative dielectric permittivity $\epsilon_{r}=10.2$ and thickness $h=0.635 \mathrm{~mm}$. For this substrate, we have generated the design graphics shown in Figs. 2 and 3. We have made use of the well-known formula relating the length $l$ of a line section of high characteristic impedance $Z_{0}$ with the value of the lumped inductance $L$ to be approximated (see [1, p. 97]) as follows:

$$
\frac{l}{L}=\frac{v_{p}}{Z_{0}}=\frac{c}{\sqrt{\epsilon_{\mathrm{eff}}} Z_{0}}
$$

where $v_{p}=c / \sqrt{\epsilon_{\mathrm{eff}}}$ is the phase velocity in the transmission line, $c$ is the speed of light in vacuum, and $\epsilon_{\text {eff }}$ is the effective dielectric constant of the transmission line. In Fig. 2, the impedance $Z_{0}^{s}$ of a transmission line consisting of a microstrip with a centered slot in the ground plane has been represented as a function of the slot width $s_{R}$ for different values of the strip width $w_{t}$. From the value of the characteristic impedance and the effective dielectric constant, the ratios $l_{i} / L_{s i}$ have been calcu- 


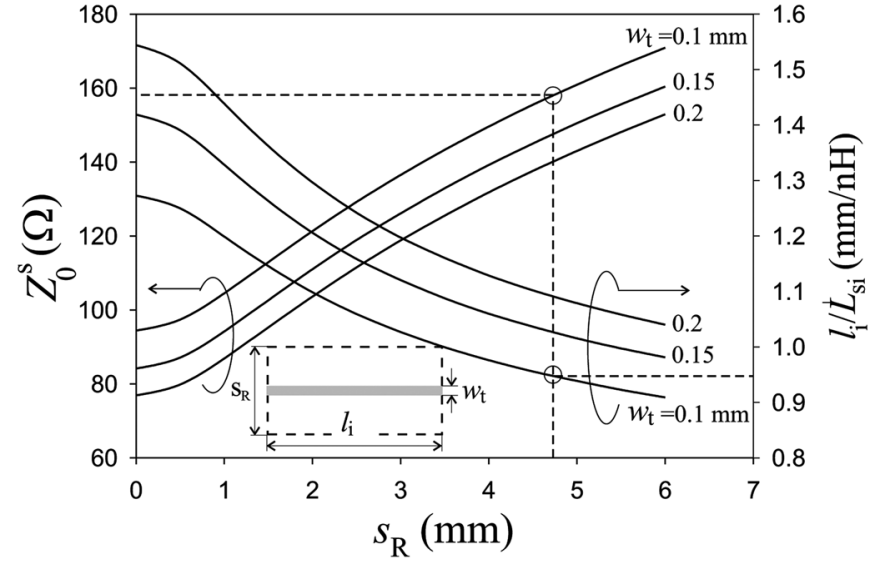

Fig. 2. Characteristic impedances $Z_{0}^{s}$ and normalized values of line section lengths $l_{i} / L_{s i}$ of a microstrip with slotted ground plane as a function of the slot width $s_{R}$ for several strip width values $\left(w_{t}\right)$.

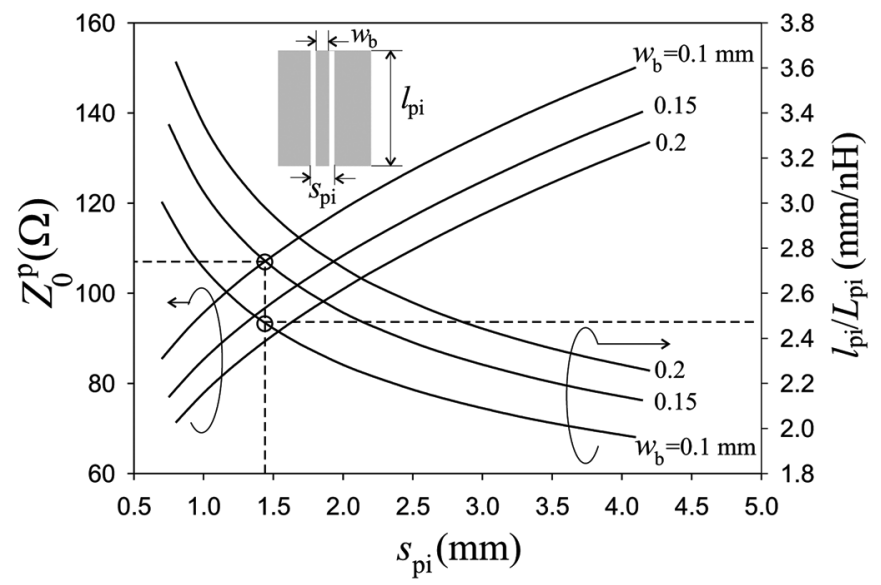

Fig. 3. Characteristic impedances $Z_{0}^{p}$ and normalized values of line section lengths $l_{p i} / L_{p i}$ of a CPW as a function of the slot width $s_{p i}$ for several values of the center strip width $\left(w_{b}\right)$.

lated using (1). As expected, for each strip-width value, the characteristic impedance is a monotonously increasing function of the slot width. Since the use of higher characteristic impedance values yields smaller values of the line lengths, some circuit size reduction is achieved using the slot (when compared with the case $s_{R}=0$, i.e., conventional microstrip lines). In Fig. 3, we have plotted the variation of the characteristic impedance of a CPW $Z_{0}^{p}$ versus the slot width $s_{p i}$ for different values of the center strip width $w_{b}$. From $Z_{0}^{p}$ and the corresponding value of $\epsilon_{\mathrm{eff}}$, we have obtained the normalized length $l_{p i} / L_{p i}$ by applying again (1), but taking into account that the inductors $L_{p i}$ are approximated by using the parallel connection of two high-impedance CPWs shorted to ground (see Fig. 1). In this way, the correct value for $l_{p i} / L_{p i}$ has to be twice the result provided by (1). As can be seen from Fig. 3, if we retain the same value of the center strip width $w_{b}$, we can get higher characteristic impedance values $Z_{0}^{p}$ and shorter line lengths $l_{p i}$ by increasing the slot width $s_{p i}$. All the results for the characteristic impedances and effective dielectric constants required to generate Figs. 2 and 3 have been computed using a fast and
TABLE III

Final GeOMEtrical Values (IN Millimeters) OF the DESIGNED AND FABRICATED FILTERS $(A$ AND $B$ )

\begin{tabular}{c|c}
\hline Filter $A$ & Filter $B$ \\
\hline & \\
$s_{R}=4.76$ & $s_{R}=4.60$ \\
$w_{t}=w_{b}=0.10$ & $w_{t}=w_{b}=0.10$ \\
$w_{c}=4.46$ & $w_{c}=4.40$ \\
$l_{1}=3.10$ & $l_{1}=4.50$ \\
$l_{2}=1.46$ & $l_{2}=2.36$ \\
$l_{3}=3.10$ & $l_{3}=7.35$ \\
$s_{p 2}=1.42, l_{p 2}=1.03$ & $l_{4}=2.30$ \\
& $l_{5}=3.93$ \\
& $s_{p 2}=0.50, l_{p 2}=1.36$ \\
& $s_{p 4}=1.42, l_{p 4}=2.79$ \\
\hline
\end{tabular}

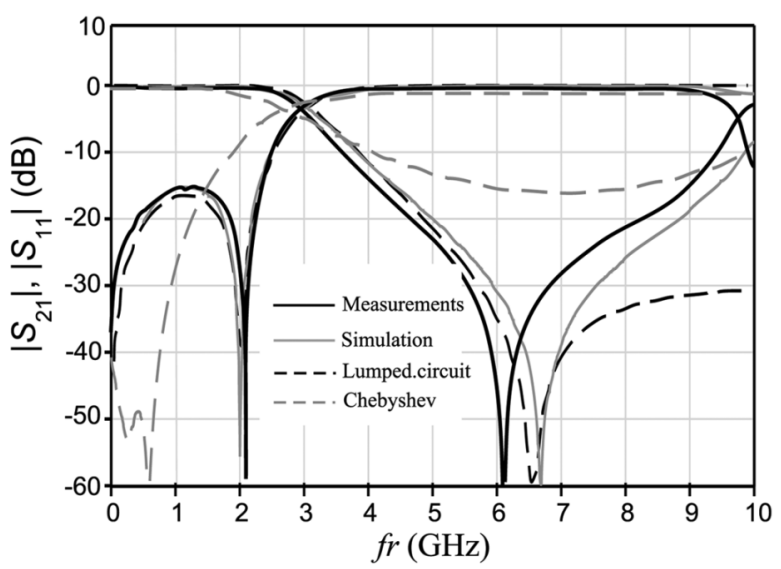

(a)

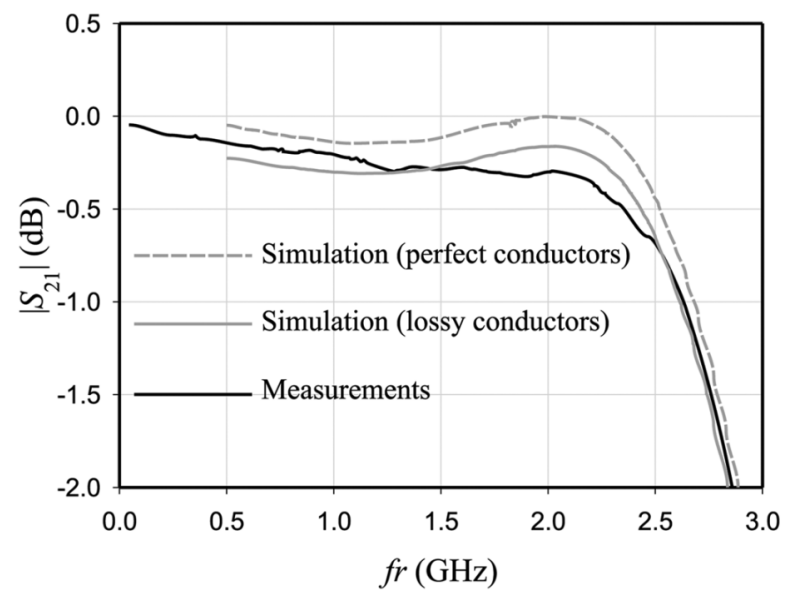

(b)

Fig. 4. (a) Electrical response of fabricated filter $A$ obtained from EM simulation (Ensemble), measurements, and lumped-circuit modeling. The simulated response of a Chebyshev A (see Table III) filter is also included. (b) Detailed view of the insertion losses in the bandpass. Measurements and simulations including and excluding ohmic losses are shown.

accurate quasi-TEM code developed in [14]. This code can handle hybrid CPW-microstrip transmission lines. The analysis in [14] uses the free surface charge distribution as unknown in the strip-like interface, whereas the electric field is used in the slot-like region. The Galerkin method in the spectral domain is then employed to solve the resulting hybrid integral equation. This suitable choice of unknown functions is one of the key points for the high numerical efficiency of the method. The other point is that drastic exponential convergence of the 


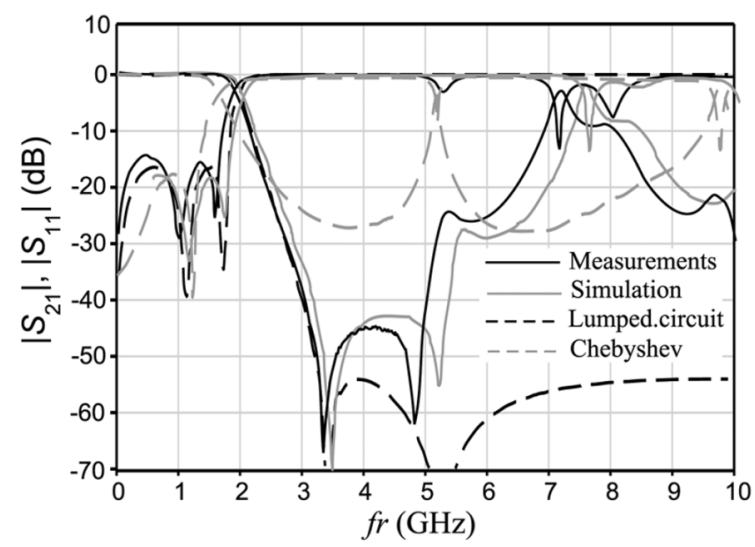

(a)

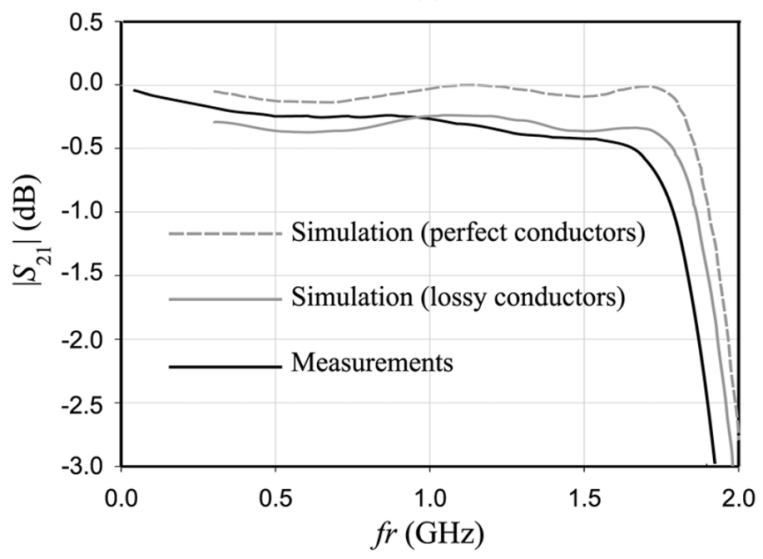

(b)

Fig. 5. (a) Electrical responses of fabricated filter $B$ obtained from EM simulation (Ensemble), measurements, and lumped-circuit modeling. The simulated response of a Chebyshev $B$ (see Table IV) filter is also included. (b) Detailed view of the insertion losses in the bandpass. Measurements and simulations including and excluding ohmic losses are shown.

spectral series is achieved by using the methods described in [15]-[17]. In conclusion, the algorithm in [14] allows us to generate the curves in Figs. 2 and 3 within a few seconds (Pentium IV PC platform).

In order to clarify the design method, below we have enumerated the steps followed to obtain the filter geometry (as an example, we use filter $A$, but the geometry of filter $B$ can be extracted in a similar way).

Step 1) Determination of the strip width $w_{t}$ and the slot width $s_{R}$ (see Fig. 1). Many different values are reasonably possible (the design is flexible at this point) provided the choice leads to a high value of the characteristic impedance $Z_{0}^{s}$ (the approximate equation (1) should yield good results). For filter $A$, we have chosen the values $w_{t}=0.1 \mathrm{~mm}$ and $s_{R}=4.76 \mathrm{~mm}$ (we have introduced a marker corresponding to those values in Fig. 2). This corresponds to $Z_{0}^{s}=158.4 \Omega$ and $l_{i} / L_{s i}=0.946 \mathrm{~mm} / \mathrm{nH}$. From the values of $L_{s i}$, shown in Table I, we obtain $l_{1}=l_{3}=3.10 \mathrm{~mm}$.

Step 2) A similar procedure has been followed when choosing $w_{b}$ and $s_{p i}$ (again, the choice of these values is arbitrary to some extent, provided high-impedance values are obtained). For filter $A$,
TABLE IV

GeOMETRICAL PARAMETERS (IN Millimeters) OF STANDARD CHEBysheV SI-LPF IN MICROSTRIP TECHNOLOGY WITH THE SAME $f_{c}$ AND $L_{A R}$ THAN FILTERS $A$ AND $B$ PROPOSED IN THIS PAPER

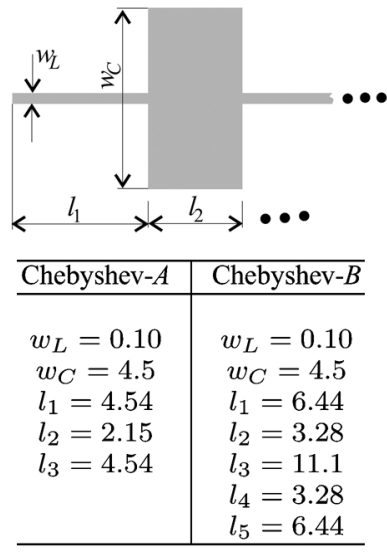

we have selected $w_{b}=0.1 \mathrm{~mm}$ and $s_{p 2}=1.42 \mathrm{~mm}$. This point has been marked in Fig. 3 to obtain $Z_{0}^{p}=106.6 \Omega$ and $l_{p i} / L_{p i}=2.48 \mathrm{~mm} / \mathrm{nH}$. From the value of $L_{p 2}$ in Table I, we get $l_{2}=1.46 \mathrm{~mm}$.

Step 3) The capacitor widths have been determined in such a way that $w_{c}$ is slightly smaller than $s_{R}$ (specifically, for filter $\left.A, w_{c}=4.46 \mathrm{~mm}\right)$. Therefore, the strips connecting the backside capacitor patches with the ground plane can be approximated as CPWs because only a very short section of these strips do not have lateral ground planes. Note that the value of $w_{c}$ has to be chosen in such a way that transverse resonances within the frequency range of interest (i.e., the bandpass and its vicinity) are precluded [1]. This condition also limits the maximum value of $s_{R}$. The length $l_{2}$ has been calculated in a first-order approximation by using the expression for the capacitance of an ideal parallel-plate capacitor. From the value of $C_{p 2}$ in Table I, we obtain the value $l_{2}=2.22 \mathrm{~mm}$. This method has been preferred to use an equation similar to (1) for the length of a low-impedance line section owing to the particular etching in the backside of the substrate. However, because of edge effects, the length obtained from the parallel-plate capacitor expression must be conveniently shortened. In this step, we have chosen to use a commercial EM simulator (in our case, Ensemble) so as to obtain this geometrical parameter of the filter. The optimization criterion has been to determine the lengths $l_{i}$ ( $i$ even) so as to make coincident the frequencies of the transmission zeros of the lumped-circuit response and those of the simulated (full-wave) filter response. Using modern EM simulators instead of approximate formulas is preferred because existing parasitics and any possible full-wave effects are automatically taken into account. In case of our filter $A$, the EM correction leads to $l_{2}=1.46 \mathrm{~mm}$.

The final geometrical parameters of filters $A$ and $B$ are shown in Table III. The total lengths of the filters are $l_{A}=\sum l_{i}=$ $7.66 \mathrm{~mm}$ and $l_{B}=22.44 \mathrm{~mm}$. The electrical responses of 


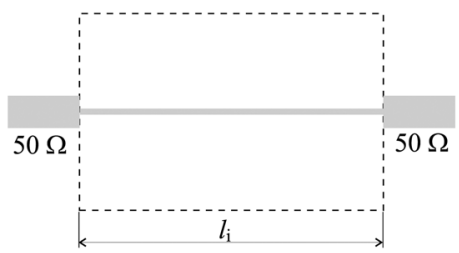

(a)

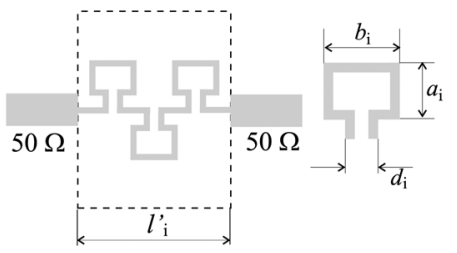

(b)

Fig. 6. (a) Straight high-impedance line section with slot in the ground plane. (b) Its equivalent meander line with series-connected open loops.

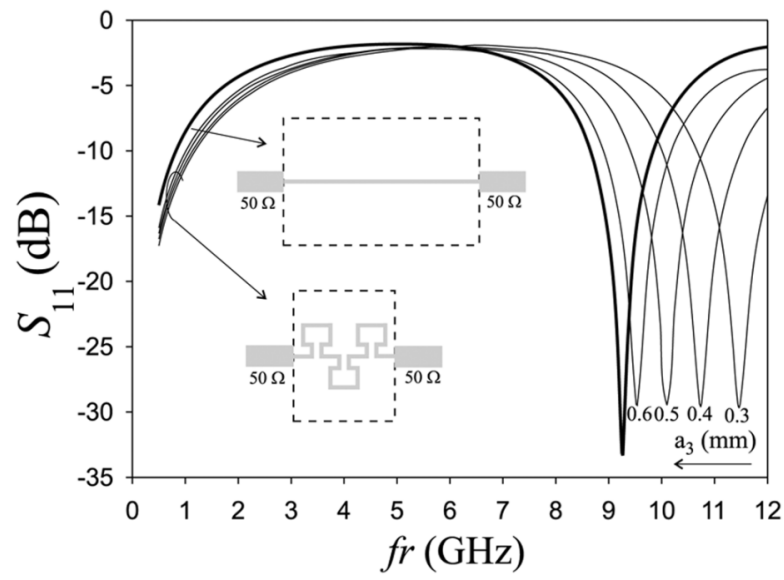

Fig. 7. Extraction of the meander geometry of Section III of the reduced size filter. Narrow lines correspond to return losses of meandered lines when $a_{3}$ is varied. The thick line is the return loss of the straight high characteristic impedance section to be substituted.

the designed filters $A$ and $B$ are shown in Figs. 4 and 5, respectively. In these figures, we compare the insertion and return losses obtained through EM simulation, measurements, and the lumped-circuit model prediction. Since the level of losses in the bandpass is very low, more detailed curves for the insertion losses are included in Figs. 4(b) and 5(b). A reasonably good agreement can be found between EM simulation and measurements (particularly good agreement is found in and around the passband). Discrepancies could be attributed to the limited accuracy of the available photoetching fabrication process. Moreover, these results agree reasonably well with those provided by the lumped-circuit model in the passband and its vicinity. Obviously, spurious bands appear at higher frequencies (above the frequencies of the transmission zeros) owing to the resonances of the transmission lines involved in the filters. To illustrate this fact, we have also included the EM responses of standard Chebyshev SI-LPFs [see Figs. 4(a) and 5(a)] with the same cutoff frequency and passband ripple designed in conventional microstrip technology. The geometrical parameters of these Chebyshev filters are shown in Table IV. Note that, in addition to the expected sharper bandpass, the section lengths of

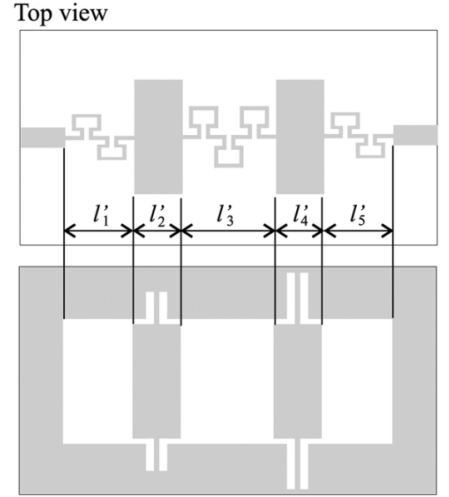

Bottom view

(a)

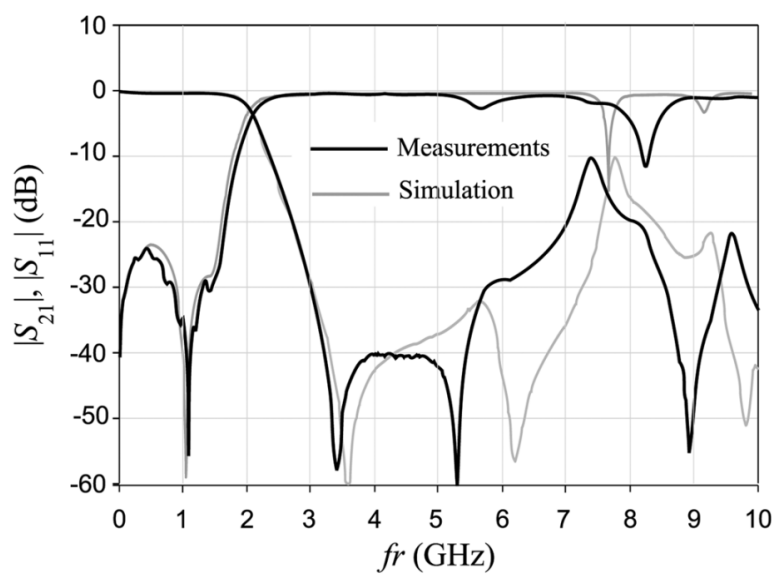

(b)

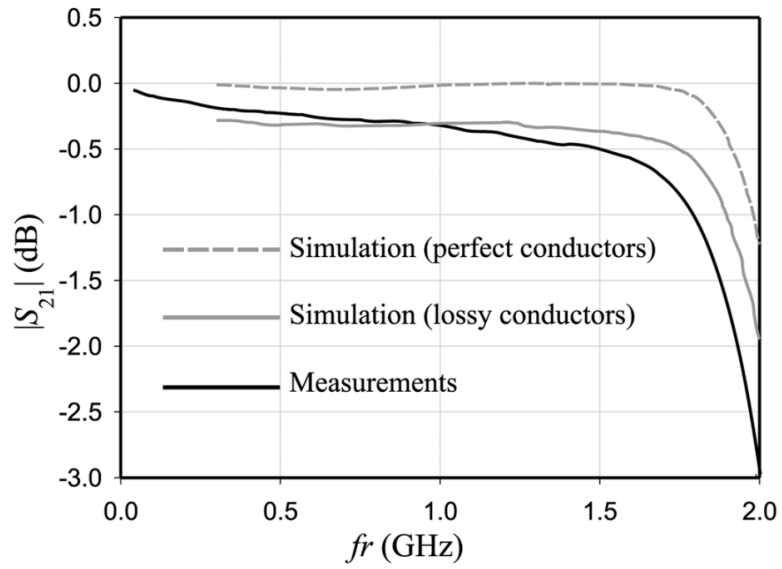

(c)

Fig. 8. (a) Layout of the miniaturized SI-LPF with the meandered lines (dimensions in the text). (b) Electrical response of filter in (a) obtained from EM simulation (Ensemble) and measurements. (c) Detailed view of the insertion losses in the bandpass. Measurements and simulations including and excluding ohmic losses are shown.

the elliptic filters are physically shorter than those of Chebyshev designs, thus achieving a significant circuit size reduction (the total length of Chebyshev filters are $l_{C A}=11.23 \mathrm{~mm}$ and $l_{C B}=30.54 \mathrm{~mm}$ ). Furthermore, it can be proved that the transmission lines involved in the elliptic filters are also electrically shorter than those of Chebyshev filters and, therefore, undesirable bands are moved to higher frequencies [11]. For instance, 
the spurious band of the Chebyshev response appearing in Fig. 5 at $5.2 \mathrm{GHz}$ (owing to the half-wave resonance of Section III) has been shifted to $7.6 \mathrm{GHz}$ in the new design.

\section{SIZE REDUCTION OF ELLIPTIC SI-LPF DESIGNS}

We have shown in Section II that a meaningful reduction of size (around 30\%) can be achieved with the new elliptic designs based on double-sided technology (see Fig. 1) with respect to Chebyshev designs implemented in conventional microstrip technology. However, additional reduction can be achieved with a little bit more effort. For instance, the total length of filter B is four times its own width (the width of the filter is defined as the slot width plus the length of the longest CPW, i.e., $s_{R}+l_{p 2}$ in the case of filter $B$ ). The goal here is to propose a shorter version of filter $B$ having the same electrical specifications. The filter length can be significantly reduced if, for instance, we substitute the straight high-impedance line sections by meandered lines built using series open loops such as those shown in Fig. 6. The geometry of the loops can be easily obtained using the EM simulator. The procedure basically consists of varying one of the geometrical parameters of the open loops until the electrical response of the single straight line (fed by $50-\Omega$ lines) to be substituted and the new meandered line become similar. As an example, the process can be visualized in Fig. 7. The thick solid line corresponds to the return loss of section 3 of filter $B$. The narrow lines correspond to the responses of meandered lines in which we have fixed $l_{3}^{\prime}=3.3 \mathrm{~mm}, b_{3}=0.8 \mathrm{~mm}$, and $d_{3}=0.4 \mathrm{~mm}$, while the value of $a_{3}$ varies from 0.3 to $0.6 \mathrm{~mm}$. The final value used is $a_{3}=0.65 \mathrm{~mm}$. The same procedure has been employed in the design of sections 1 and 5 (final meander line values are $l_{1}^{\prime}=1.5 \mathrm{~mm}, b_{1}=0.8 \mathrm{~mm}, d_{1}=0.4 \mathrm{~mm}$, $a_{1}=0.5 \mathrm{~mm} ; l_{5}^{\prime}=1.5 \mathrm{~mm}, b_{5}=0.8 \mathrm{~mm}, d_{5}=0.4 \mathrm{~mm}$, $a_{5}=0.4 \mathrm{~mm}$ ). Sections 2 and 4 remain unchanged. The final design has a total length of $l=11 \mathrm{~mm}$, i.e., a $50 \%$ smaller than filter $B$ designed in the previous section. It must be pointed out that a coupling capacitive effect appears between the patches of sections 2 and 4 because of the reduction of $l_{3}$ and, therefore, the filter response slightly differs from that of filter $B$. These discrepancies become more important when the length $l_{3}$ decreases. Fig. 8 shows the EM simulation response and measurements of the miniaturized SI-LPF filter (a detailed view of the insertion losses in the bandpass is included again). Reasonably good agreement is found between both results, particularly at the low-frequency portion of the measured spectrum. As mentioned before, a small displacement of the transmission zeros can be observed when compared with the response of filter $B$ shown in Fig. 5.

\section{CONCLUSIONS}

In this paper, we have presented a new elliptic response SI-LPF filter with a sharp bandpass and reduced size. The design method is based on the use of the double-sided MIC technology to build the different components of the filter. The impedance of the high-impedance sections has been increased by using slots in the ground plane. The low-impedance sections have been approximated as parallel-plate capacitors. The new idea is that the patch of the capacitor in the backside of the substrate is connected to the ground plane by means of high-impedance CPW lines, which act as inductors and yield the transmission zeros of the elliptic design. The lengths of the capacitors have been tuned using the EM simulator Ensemble in order to match the transmission zeros to those provided by the lumped equivalent-circuit model. Although the size of the new filters is meaningfully smaller than the size of the corresponding standard Chebyshev filters in microstrip technology, an additional improvement has been introduced in order to reduce the filter size. In this way, the straight high characteristic-impedance series-connected line sections have been substituted by meandered lines with series-connected open loops. The EM simulator has been used to calculate the dimensions of the meandered lines and, finally, a miniaturized modified version of the elliptic SI-LPF has been obtained. An additional reduction above $50 \%$ in the length of the filters has been then finally carried out.

\section{REFERENCES}

[1] J. S. Hong and M. J. Lancaster, Microstrip Filters for RF/Microwave Applications. New York: Wiley, 2001.

[2] A. D. Abdel-Rahman, A. K. Verma, A. Boutejdar, and A. S. Omar, "Control of bandstop response of hi-lo microstrip low-pass filter using slot in ground plane," IEEE Trans. Microw. Theory Tech., vol. 52, no. 3, pp. 1008-1013, Mar. 2004.

[3] J. S. Lim, C. S. Kim, D. Ahn, Y. C. Jeong, and S. Nam, "Design of low-pass filters using defected ground-plane structure," IEEE Trans. Microw. Theory Tech., vol. 53, no. 8, pp. 2539-2545, Aug. 2005.

[4] J. Carcía-Carcía, J. Bonache, F. Falcone, J. D. Baena, F. Martìn, I. Gil, T. Lopetegui, M. A. G. Laso, A. Marcotegui, R. Marqués, and M. Sorolla, "Stepped-impedance lowpass filters with spurious passband suppression," Electron. Lett., vol. 40, pp. 881-882, Jul. 2004.

[5] S. A. Alseyab, "A novel class of generalized Chebyshev low-pass prototype for suspended substrate stripline filters," IEEE Trans. Microw. Theory Tech., vol. MTT-30, no. 9, pp. 1341-1347, Sep. 1982.

[6] F. Giannini, M. Salerno, and R. Sorrentino, "Design of low-pass elliptic filters by means of cascaded microstrip rectangular elements," IEEE Trans. Microw. Theory Tech., vol. MTT-30, no. 9, pp. 1348-1353, Sep. 1982.

[7] L. H. Hsieh and K. Chang, "Compact elliptic-function low-pass filters using microstrip stepped-impedance hairpin resonators," IEEE Trans. Microw. Theory Tech., vol. 51, no. 1, pp. 193-199, Jan. 2001.

[8] W. Tu and K. Chang, "Compact microstrip low-pass filter with sharp rejection," IEEE Microw. Wireless Compon. Lett., vol. 15, no. 6, pp. 404-406, Jun. 2005.

[9] M. Aikawa and H. Ogawa, "Double-sided MIC's and their applications," IEEE Trans. Microw. Theory Tech., vol. 37, no. 2, pp. 406-413, Feb. 1989.

[10] L. Zhu, H. Bu, K. Wu, and M. Stubbs, "Unified CAD model of microstrip line with backside aperture for multilayered integrated circuit," in IEEE MTT-S Int. Microw. Symp. Dig., Jun. 2000, vol. 2, pp. 981-984.

[11] S. Sun and L. Zhu, "Stopband-enhanced and size-miniaturized lowpass filters using high-impedance property of offset finite-ground microstrip line," IEEE Trans. Microw. Theory Tech., vol. 53, no. 9, pp. 2844-2850, Sep. 2005.

[12] M. C. Velázquez, J. Martel, and F. Medina, "Parallel coupled microstrip filters with ground-plane aperture for spurious band suppression and enhanced coupling," IEEE Trans. Microw. Theory Tech., vol. 52, no. 3, pp. 1082-1086, Mar. 2004.

[13] — "Parallel coupled microstrip filters with floating ground-plane conductor for spurious-band suppression," IEEE Trans. Microw. Theory Tech., vol. 53, no. 5, pp. 1823-1828, May 2005.

[14] J. Martel and F. Medina, "A suitable integral equation for the quasi-TEM analysis of hybrid strip/slot-like structures," IEEE Trans. Microw. Theory Tech., vol. 49, no. 1, pp. 224-228, Jan. 2001.

[15] F. Medina and M. Horno, "Quasi-analytical static solution of the boxed microstrip line embedded in a layered medium," IEEE Trans. Microw. Theory Tech., vol. 40, no. 9, pp. 1748-1756, Sep. 1992.

[16] E. Drake, F. Medina, and M. Horno, "Improved quasi-TEM spectral domain analysis of boxed coplanar multiconductor microstrip lines," IEEE Trans. Microw. Theory Tech., vol. 41, no. 2, pp. 260-267, Feb. 1993. 
[17] —, "Quick computation of $[C]$ and $[L]$ matrices of generalized multiconductor coplanar waveguide transmission lines," IEEE Trans. Microw. Theory Tech., vol. 42, no. 12, pp. 2328-2335, Dec. 1994.

[18] H. Peddibhotia and R. K. Settagori, "Compact folded line bandstop and low pass filters," Microw. Opt. Technol. Lett., vol. 42, pp. 44-46, Jul. 2004.

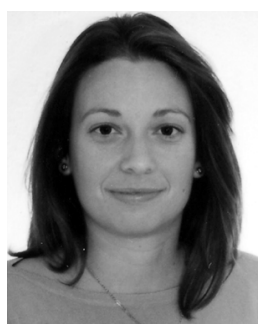

María del Castillo Velázquez-Ahumada was born in Lebrija, Sevilla, Spain, in 1976. She received the Licenciado degree in physics from the Universidad de Sevilla, Seville, Spain, in 2001, and is currently working toward the Ph.D. degree in electronics and electromagnetism at the Universidad de Sevilla.

Her research focus is on printed passive microwave filters and couplers.

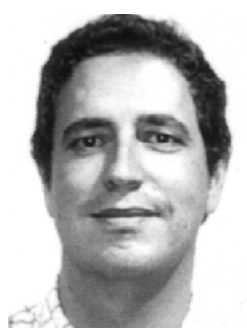

Jesús Martel was born in Seville, Spain, in 1966. $\mathrm{He}$ received the Licenciado and Doctor degrees in physics from the Universidad de Sevilla, Seville, Spain, in 1989 and 1996, respectively.

Since 1992, he has been with the Departamento de Física Aplicada II, Universidad de Sevilla, where in 2000, he became an Associate Professor. His current research interest is focused on the numerical analysis of planar transmission lines, modeling of planar microstrip discontinuities, design of passive microwave circuits, microwave measurements, and

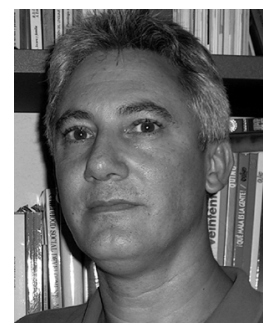

Francisco Medina (M'90-SM'01) was born in Puerto Real, Cádiz, Spain, in 1960. He received the Licenciado (with honors) and Doctor degrees in physics from the Universidad de Sevilla, Seville, Spain, in 1983 and 1987 respectively.

From 1985 to 1989, he was an Assistant Professor with the Departamento de Electrónica y Electromagnetismo, Universidad de Sevilla, where since 1990, he has been an Associate Professor. Since 1998, he has been the Head of the Grupo de Microondas, Departamento de Electrónica y Electromagnetismo, Universidad de Sevilla. His research interest includes analytical and numerical methods for guiding, resonant, and radiating planar structures, passive planar circuits, periodic structures, and the influence of anisotropic materials (including microwave ferrites) on such systems. He is also interested in artificial media modeling and design. He is a member of the Editorial Board of the International Journal of RF and Microwave Computer-Aided Engineering.

Dr. Medina has been a member of the Technical Programme Committees (TPCs) of several national and international conferences. He has been a reviewer for a number of IEEE, Institution of Electrical Engineers (IEE), U.K., and American Physics Association journals. He is a member of the Massachusetts Institute of Technology (MIT) Electromagnetics Academy. He was the recipient of two research scholarships presented by the Spanish Ministerio de Educación y Ciencia (MEC) (1983) and the Spanish MEC/French Ministère de la Recherche et la Technologie (ENSEEIHT, Toulouse, France) (1986).

artificial media. 